\title{
Correction to: Cognitive penetration and the cognition-perception interface
}

\section{Daniel C. Burnston ${ }^{1}$}

Published online: 4 October 2018

(c) Springer Nature B.V. 2018

\section{Correction to: Synthese (2017) 194:3645-3668 \\ https://doi.org/10.1007/s11229-016-1116-y}

On page 3653, there is a mistake in the explanation of the Cornsweet illusion. In fact, the explanation is that the panel perceived as darker is facing towards the light source - in the case of this figure the light is coming from the right. If this were the case, then the panel on the right would be more brightly illuminated, and the patch on the left more dimly illuminated. Thus, in order for the right panel to produce the same luminance value at the retina as the left panel, it must in fact be darker, and the visual system represents it so. The author apologizes for this error, but it does not change the salient points of the example for the succeeding discussion.

The original article can be found online at https://doi.org/10.1007/s11229-016-1116-y.

Daniel C. Burnston

dburnsto@tulane.edu

1 Department of Philosophy, Tulane University, 105 Newcomb Hall, New Orleans, LA 70118, USA 